\title{
Comunicación para la prevención de arbovirosis: adecuación de iniciativas de la OPS al contexto cubano
}

\author{
Yisel Hernández, ${ }^{1}$ Marta Castro, ${ }^{1}$ Susett Pérez, ${ }^{2}$ Alina Pérez, ${ }^{3}$ \\ Linda S. Lloyd ${ }^{4}$ y Dennis Pérez ${ }^{1}$
}

Forma de citar Hernández Y, Castro M, Pérez S, Pérez A, Lloyd LS, Pérez D. Comunicación para la prevención de arbovirosis: adecuación de iniciativas de la OPS al contexto cubano. Rev Panam Salud Publica. 2018;42:e146. https://doi.org/10.26633/RPSP.2018.146

\begin{abstract}
RESUMEN El presente trabajo tiene como objetivo describir las oportunidades y los desafíos de los procesos de capacitación en la articulación de iniciativas regionales y nacionales de comunicación para la prevención de arbovirosis, usando como ejemplo las lecciones aprendidas en el contexto cubano. En febrero de 2016 y diciembre de 2017 profesionales del Instituto de Medicina Tropical Pedro Kourí participaron en la realización de talleres de fortalecimiento de capacidades de comunicación para la prevención de arbovirosis en la Atención Primaria de Salud con la incorporación paulatina de los más recientes enfoques promovidos por la Organización Panamericana de la Salud sobre el tema. Se capacitaron especialistas de diferentes perfiles profesionales a nivel nacional, provincial, municipal y de áreas de salud, se formaron y consolidaron equipos multidisciplinarios en los diferentes niveles, se asignaron y articularon tareas concretas a cada nivel, y se establecieron sitios de demostración de los enfoques de comunicación promovidos en los talleres. Asimismo, se identificaron necesidades de aprendizaje en comunicación social y se incorporaron a la estrategia nacional 2018-2021 actividades de capacitación del personal técnico y de monitoreo y evaluación con el objetivo de garantizar la implementación de acciones de comunicación y su sostenibilidad. No obstante, las instituciones participantes tendrán que afrontar desafíos propios de la comunicación en la prevención de las arbovirosis como, por ejemplo, la fluctuación del personal entrenado, la movilización de recursos para la comunicación, y la identificación de indicadores adecuados de evaluación.
\end{abstract}

Palabras clave Comunicación social; Aedes aegypti; infecciones por arbovirus; control de vectores; Cuba.

Centro de Investigaciones, Diagnóstico y Referencia (CIDR), Instituto de Medicina Tropical Pedro Kourí (IPK), La Habana, Cuba. La correspondencia se debe dirigir a Yisel Hernández. Correo electrónico: yhbarrios@ipk.sld.cu

2 Departamento de Promoción de Salud, Unidad de Promoción de Salud y Prevención de Enfermedades (Prosalud), La Habana, Cuba.

3 Enfermedades Transmisibles y Determinantes Ambientales de Salud (CDE-VT), Organización Panamericana de la Salud, La Habana, Cuba.

4 Grupo Técnico-Dengue Internacional, Programa Regional de Dengue, Organización Panamericana de la Salud, Washington DC, Estados Unidos de América.
El escenario actual de la Región de las Américas se caracteriza por la emergencia y cocirculación de varias arbovirosis (1). Dentro de este grupo, las enfermedades transmitidas principalmente por Aedes aegypti (Diptera: Culicidae), que incluyen dengue, zika, chikungunya y fiebre amarilla, se han asociado con el desarrollo de diversas alternativas para atenuar su impacto sanitario, económico, político y social (2-4).
La comunicación, como fenómeno que se extiende a todas las esferas de la vida, y como componente esencial de muchas intervenciones en salud, cobra una importancia significativa en los programas de control de Aedes aegypti (5-6). En consecuencia, las acciones tradicionales de prevención de arbovirosis (control químico, físico y biológico del vector) se complementan con estrategias de comunicación con diferentes propósitos: aumentar los 
conocimientos de la población, fomentar la participación comunitaria en acciones de prevención y control, y promover cambios en los comportamientos de riesgo (7-9).

Entre los esfuerzos de la Organización Panamericana de la Salud (OPS) que avalan el desarrollo de la comunicación en este campo se destacan la Estrategia de Gestión Integrada para la prevención y el control del dengue (EGI-dengue) y la metodología de Comunicación para el Impacto Conductual (COMBI). En la EGI-dengue se propuso un modelo de gestión integrada que incluyó inicialmente la comunicación social como componente esencial (5). La metodología COMBI reemplaza estrategias anteriores de información, educación y comunicación (IEC) sobre actitudes y conocimientos y se orienta a la promoción de objetivos conductuales relacionados con el control de Aedes aegypti (10-11).

La experiencia acumulada con la implementación de estas propuestas y los cambios que se produjeron en el escenario epidemiológico internacional han contribuido a que la comunicación se considere eje transversal de las acciones de prevención y control integrado de arbovirosis, a que se promueva la elaboración de mensajes sobre eliminación de criaderos principales y la búsqueda oportuna de asistencia médica, y a la utilización de la comunicación médicopaciente con este fin (12-14). Sin embargo, la materialización y el impacto de estas propuestas requieren necesariamente su articulación con las iniciativas nacionales y su adaptación a las particularidades de los sistemas de salud y sus programas, a la situación epidemiológica, a las características socioculturales, así como a las necesidades de aprendizaje de la población y del personal técnico.

En el presente trabajo se describen las oportunidades y los desafíos que plantea el proceso de fortalecimiento de las capacidades de comunicación para la prevención de arbovirosis en la articulación de iniciativas regionales y nacionales, utilizando como ejemplo algunas lecciones aprendidas como parte de este proceso en el contexto cubano.

\section{Fortalecimiento de capacidades de comunicación para la prevención de arbovirosis en Cuba}

En Cuba existe un Programa Nacional de Control de Aedes aegypti y Aedes albopictus (PCAa), que está estructurado verticalmente de acuerdo con los niveles del Sistema Nacional de Salud (SNS): nacional, provincial, municipal y atención primaria (15). El PCAa se creó en respuesta a la epidemia de dengue hemorrágico de 1981. Las modificaciones que se introdujeron en este Programa se encaminaron fundamentalmente a mejorar su rendimiento y desempeño en relación con la selección, la capacitación y la gestión de los recursos humanos, el control de la calidad, los métodos de vigilancia y control de vectores, la aplicación de la legislación sanitaria, la comunicación social, y la participación comunitaria e intersectorial. Además, tuvieron como propósito adecuar las acciones realizadas a los resultados científicos, a los cambios ambientales, económicos, políticos y socioculturales ocurridos en el país, y a la situación epidemiológica nacional e internacional del dengue (15-16).

A pesar de la voluntad política del Gobierno cubano, a finales de los años noventa el PCAa afrontó dificultades para mantener los resultados alcanzados en décadas anteriores: disminución de los índices de infestación del vector y cese de la transmisión autóctona de la enfermedad. La epidemia de dengue ocurrida en Santiago de Cuba (1997) y los subsiguientes brotes han sido el resultado de la interrelación de factores ambientales, socioeconómicos y culturales tales como: los servicios públicos de abastecimiento de agua y saneamiento limitados como consecuencia de la crisis económica de los noventa, las prácticas de riesgo de la población, el aumento del número de viajeros procedentes de países endémicos, el incremento de la susceptibilidad de la población a la infección primaria y secundaria por dengue (ausencia de circulación de virus durante 15 años), y la proliferación de Aedes aegypti por la limitada eficacia de los métodos de control disponibles en el país. Muchos de estos factores persisten en la actualidad y adquieren mayor connotación con el riesgo de introducción y transmisión autóctona de fiebre amarilla y otras arbovirosis emergentes en la Región (Chikungunya en 2014 y Zika en 2015) (15-17). Por ello, es necesario desarrollar estrategias intersectoriales e interdisciplinarias que contribuyan a su abordaje integrado (12-14). Entre las posibles estrategias, las acciones de comunicación cobran mayor importancia y visibilidad en tanto facilitan la adecuación de los diferentes métodos de control a las necesidades locales y a las especificidades de cada enfermedad, y porque pueden impactar en la movilización de recursos, en el cambio de comportamientos y en el compromiso de las comunidades con las acciones de prevención y control.

El abordaje de la comunicación social para la prevención de arbovirosis en Cuba se hace eco de los esfuerzos regionales. Sin embargo, su evolución tiene particularidades y se manifiesta en dos áreas fundamentales: en la de la investigación y en la de la implementación. En la primera, la comunicación usualmente se aborda como medio de divulgación de acciones intersectoriales y como una herramienta para el empoderamiento comunitario en el control de Aedes aegypti (17). En ella se observa una incorporación paulatina de la comunicación para el cambio conductual dirigido a la eliminación de criaderos principales (17-18). La implementación de acciones de comunicación se caracteriza por el predominio de un enfoque transmisivo que tiene como metas aumentar el conocimiento, así como la colaboración y la movilización de las comunidades en las acciones del PCAa. (15). No obstante, en los últimos años se aprecia con mayor intensidad la inclusión de enfoques teóricometodológicos como, por ejemplo, de comunicación de riesgo, de comunicación para la salud, de comunicación educativa, y de comunicación para el desarrollo. Además, se incorporan acciones y productos comunicativos que abordan la prevención y el control integrado de las arbovirosis. Sin embargo, los esfuerzos por incorporar los enfoques de comunicación para la prevención de arbovirosis promovidos por la OPS datan de fechas relativamente recientes.

\section{Taller de planificación para impactar la conducta (COMBI) en la prevención de las enfermedades transmitidas por vectores}

En Cuba, uno de los primeros acercamientos a las propuestas de la OPS mencionadas anteriormente fue el Taller COMBI, que se celebró en febrero de 2016. Coordinado por el Ministerio de Salud Pública (MINSAP), la Unidad de Promoción de Salud y Prevención de Enfermedades (Prosalud), el Instituto de Medicina Tropical Pedro Kourí (IPK) y la OPS, el taller se convirtió en un escenario para articular las experiencias cubanas 
en comunicación, las lecciones aprendidas en el área de la investigación y las propuestas de la OPS para fortalecer y visibilizar el desarrollo de la comunicación en este campo.

El taller tuvo como objetivo fortalecer las capacidades del SNS en la planificación y ejecución de acciones de comunicación y participación social para la prevención de arbovirosis con resultados medibles en los cambios conductuales producidos. Para ello, se realizó una capacitación en cascada en la cual se formaron dos equipos nacionales $\mathrm{y}$ tres provinciales (La Habana, Villa Clara y Santiago de Cuba) integrados por epidemiólogos, entomólogos, investigadores sociales y especialistas en promoción de la salud. Los equipos provinciales en coordinación con los nacionales replicarían la capacitación en el resto de las provincias del país hasta llegar al nivel municipal. A pesar de los esfuerzos realizados, la capacitación no incluyó la Atención Primaria de Salud (APS), que es donde se materializan las acciones de prevención y control. Como consecuencia, no se generaron acciones concretas en las comunidades.

Las lecciones aprendidas pusieron de manifiesto la necesidad de construir capacidades no solo en los niveles gerenciales del PCAa, donde se generan los procedimientos estandarizados que pautan las acciones de prevención y control, sino también en médicos y enfermeras de la familia, en técnicos de control de vectores y en especialistas en promoción de salud que implementan estos procedimientos en la APS.

\section{Taller de fortalecimiento de capacidades de comunicación para la prevención de arbovirosis en la APS}

En diciembre de 2017, investigadores del IPK organizaron el Taller de Fortalecimiento de Capacidades de Comunicación para la Prevención de Arbovirosis en la APS bajo el auspicio del MINSAP y de la OPS. Se seleccionó el municipio La Lisa porque presenta características promedio con relación a otros municipios de La Habana en cuanto a riesgos entomológicos, epidemiológicos, ambientales y socioculturales para la transmisión de arbovirosis. Se capacitaron 45 profesionales de salud vinculados con la prevención y el control de arbovirosis: decisores de Prosalud, de la Dirección Provincial de Higiene y Epidemiología de La
Habana, y de la Dirección de Salud del municipio La Lisa, así como especialistas en promoción de salud, epidemiólogos, médicos y enfermeras de la familia, técnicos del programa de control de vectores, y otros especialistas de las cinco áreas de salud del municipio.

El taller tuvo una duración de 64 horas (32 presenciales y 32 de trabajo en el terreno) y se sustentó en dos principios metodológicos de probada efectividad en el empoderamiento comunitario en Cuba: la construcción colectiva (dialógica) de conocimientos y la lógica prácticateoría-práctica transformada (19). La práctica tuvo el propósito de comprobar el nivel de conocimiento empírico de los participantes en el tema e identificar aciertos y desaciertos. Para ello, se presentaron la situación epidemiológica y las experiencias de comunicación en el municipio, estudios previos de conocimientos, percepciones y prácticas de la población sobre prevención de arbovirosis y control de su principal vector, y generalidades de estrategias, campañas y productos nacionales de comunicación en este campo.

Durante la teorización, el conocimiento empírico se cotejó con el contenido temático del taller: la evolución de las estrategias de comunicación y movilización social en programas de control de Aedes aegypti, el enfoque de comunicación para el cambio conductual, el monitoreo y la evaluación de acciones de comunicación, el enfoque de comunicación centrada en la persona (20), así como los resultados de investigadores del IPK sobre la gestión de comunicación comunitaria, la utilidad de los sistemas de información geográfica, la plasticidad ecológica del vector $\mathrm{y}$ sus implicaciones en las

\section{CUADRO 1. Resultados del proceso de fortalecimiento de capacidades de comunicación para la prevención de arbovirosis en Cuba, febrero 2016-diciembre 2017}

Capacitación de especialistas de diferentes perfiles profesionales en los niveles gerenciales y de implementación del Sistema Nacional de Salud

Definición de tareas concretas en todos los niveles y articulación entre ellos para facilitar la concreción de acciones, planes y estrategias de comunicación comunitarias

Conformación y consolidación de equipos multidisciplinarios en los diferentes niveles del Sistema Nacional de Salud con experiencia en la elaboración de planes COMBI

Establecimiento de sitios de demostración del enfoque de comunicación para el cambio conductual orientado a criaderos principales y centrado en la persona

Inclusión de un programa de capacitación dirigido a médicos, enfermeras y técnicos de control de vectores de la APS para la distribución de mensajes específicos orientados hacia la conducta deseada desde su experiencia y relación con las personas, familias o comunidades

Inclusión en la Estrategia Nacional de Comunicación 2018-2021 de actividades de capacitación del personal técnico, de monitoreo y de evaluación de acciones de comunicación comunitaria

Fuente: elaborado por las autoras con datos de los talleres. 
recursos, la participación de especialistas del equipo de comunicación en espacios de toma de decisiones, la identificación de indicadores adecuados de evaluación, y la revalorización por parte de los decisores del PCAa del papel y del tiempo de las acciones de comunicación en la prevención y el control de arbovirosis.

\section{Conclusiones}

Los procesos de fortalecimiento de las capacidades facilitan el diálogo y la adaptación de las iniciativas de la OPS a la planificación y la implementación de acciones de comunicación para la prevención de arbovirosis en los contextos nacionales y locales. Se recomienda que los programas de control de Aedes aegypti de los diferentes países de la Región incluyan la construcción de capacidades en

1. Pan American Health Organization. Population exposed to arbovirus risk factors in the Americas. Washington, DC: PAHO; 2018. Disponible en: http://ais. paho.org/phip/viz/ed_popatrisk_arbovirus.asp Acceso el 22 de enero de 2018

2. Organización Panamericana de la Salud. Estrategia para la prevención y el control de las enfermedades arbovirales. 55. ${ }^{\circ}$ Consejo Directivo, $68 .^{\text {a }}$ sesión del Comité Regional de la OMS para las Américas. Washington, DC: OPS; 2016. Disponible en: http://www.paho.org/ Acceso el 22 de enero de 2018.

3. Organización Panamericana de la Salud. Estrategia de gestión integrada para la prevención y el control de las enfermedades arbovirales. Bucaramanga: OPS; 2016.

4. Organización Mundial de la Salud. Respuesta mundial para el control de vectores 2017-2030. Documento de contexto para informar las deliberaciones de la Asamblea Mundial de la Salud en su 70. ${ }^{a}$ Reunión. Versión 5.4. Ginebra: OMS: 2017.

5. Organización Panamericana de la Salud. Estrategia de gestión integrada para la prevención y el control del dengue en Las Américas. $44 .^{\circ}$ Consejo Directivo, 55. ${ }^{\mathrm{a}}$ sesión del Comité Regional de la OMS para las Américas. Washington, DC: OPS; 2003. Disponible en: http://iris.paho.org/ xmlui/bitstream/h Acceso el 22 de enero de 2018.

6. Organización Panamericana de la Salud. Últimos adelantos técnicos en la prevención y el control del dengue en la Región de las Américas. Washington, DC: OPS; 2014. Disponible en: http://www.paho. org/ Acceso el 22 de enero de 2018.

7. World Health Organization. Global strategy for dengue prevention and control, 2012-2020. Geneva: WHO; 2012. la APS como pilar fundamental de los procesos de comunicación, así como la movilización de recursos a tales efectos.

Agradecimiento. Los autores agradecen al Ministerio de Salud Pública de Cuba y a la Organización Panamericana de la Salud su asesoramiento técnico y el acompañamiento metodológico en el desarrollo de los talleres. También quieren destacar la colaboración de especialistas del Instituto de Medicina Tropical Pedro Kourí (IPK), de la Unidad de Promoción de Salud y Prevención de Enfermedades (Prosalud) y de la Dirección Municipal de Higiene y Epidemiología del Municipio La Lisa, que se involucraron en las actividades específicas de capacitación y, en especial, la de la Dra. María del Carmen Marquetti, la Dra. Mildred González, el Lic. Jorge Pulido y el Dr. Vladimir Polo.

\section{REFERENCIAS}

Disponible en: http:/ /www.who.int/denguecontrol/9789241504034/en/ Acceso el 23 de enero de 2018.

8. World Health Organization. Handbook for integrated vector management. Geneva: WHO; 2012. Disponible en: http: / / apps.who.int/iris / bitstre am/10665/44768/1/9789241502801_eng. pdf Acceso el 8 de febrero de 2018.

9. Menjívar EV, Espinoza E, Robles JO. Estrategia de información, educación y comunicación para el abordaje del dengue, chikungunya y zika. San Salvador: Ministerio de Salud; 2015.

10. Parks W, Lloyd LS. Planificación de la movilización y comunicación social para la prevención y el control del dengue. Guía paso a paso. Ginebra: Organización Mundial de la Salud; 2004. Disponible en: http://www. ops.org.bo/ Acceso el 22 de enero de 2018.

11. Elder JP. Evaluation of communication for behavior impact (COMBI) efforts to control Aedes aegypti breeding sites in six countries. Tunis: WHO Mediterranean Centre for Vulnerability Reduction; 2005.

12. Organización Panamericana de la Salud. Taller regional para la revisión y ajuste de la "Estrategia para la prevención y el control de las enfermedades arbovirales" en las Américas. Guatemala: OPS; 2017. Disponible en: https://www.paho.org/ hq/index.php?option=com_topics\&view$=$ readall\&cid $=5521 \&$ Itemid $=40734 \& 1-$ ang=es Acceso el 7 de junio de 2018.

13. Pan American Health Organization. Integrated management strategy for dengue prevention and control in the Region of the Americas. Washington, DC: PAHO; 2018. Disponible en: http://iris.paho.org/ xmlui/handle/123456789/34860 Acceso el 7 de junio de 2018.

14. Pan American Health Organization. $55^{\text {th }}$ Directing Council, 68 $8^{\text {th }}$ Session of the Regional Committee of WHO for the
Financiación. Este estudio ha recibido financiación procedente de los Fondos Regulares para la Asistencia Técnica en el Fortalecimiento de Capacidades para la Vigilancia, Prevención y Control de Enfermedades Trasmitidas por Vectores, Bienio 2016-2017, de la Organización Panamericana de Salud.

Conflictos de interés. Linda S. Lloyd declara que fue consultora de la OPS en la preparación de los materiales "Comunicación centrada en el paciente". Las demás autoras declaran no tener conflictos de interés.

Declaración. Las opiniones expresadas en este manuscrito son responsabilidad de las autoras y no reflejan necesariamente los criterios ni la política de la RPSP / PAJPH y/o de la OPS.
Americas. Strategy for arboviral disease prevention and control. Washington, DC: PAHO; 2018. Disponible en: https:// www.paho.org/hq/dmdocuments/2016/ CD55-16-e.pdf Acceso el 6 de agosto de 2018.

15. Ministerio de Salud Pública. Programa de Vigilancia y Lucha Antivectorial. La Habana: Encimed; 2013.

16. Conner MA. Cuba Confronts Zika: All Hands on Deck. MEDICC Review. 2016; 18(1-2):6-10.

17. Pérez D, Castro M, Álvarez AM, Sánchez L, Toledo ME, Matos D, et al. Traslación a la práctica de estrategias de empoderamiento en la prevención del dengue: facilitadores y barreras. Rev Panam Salud Publica. 2016;39(2):93-100.

18. Hernández Y. Gestión de comunicación comunitaria para la prevención de arbovirosis. Propuestas teórico-metodológicas y prácticas para su fortalecimiento en el contexto cubano. [Tesis de Maestría]. La Habana: Universidad de la Habana, Facultad de Comunicación Social; 2017.

19. Sánchez L, Pérez D, Alfonso L, Castro M Sánchez LM, Van der Stuyft P, et al. Estrategia de educación popular para promover la participación comunitaria en la prevención del dengue. Rev Panam Salud Publica. 2008;24(1):61-9.

20. Forguione VP. Comunicación entre médico y paciente: más allá de una consulta, un proceso educativo. Med UIS. 2015; 28(1):7-13.

Manuscrito recibido el 8 de marzo de 2018. Aceptado para su publicación, tras revisión, el 28 de junio de 2018. 
ABSTRACT

\section{Communication for} arboviral disease prevention: tailoring PAHO initiatives to the Cuban context

Keywords Social communication; Aedes aegypti; arbovirus infections; vector control; Cuba.
The purpose of this paper is to describe the opportunities and challenges of training processes in the coordination of regional and national communication initiatives for arboviral disease prevention, using the lessons learned in the Cuban context as an example. In February 2016 and December 2017, professional staff from the Pedro Kourí Institute of Tropical Medicine attended workshops for strengthening communication capacities for arboviral disease prevention in primary health care settings through gradual introduction of the most recent approaches promoted by the Pan American Health Organization in this regard. National, provincial, municipal, and health area specialists with diverse professional profiles were trained; multidisciplinary teams were strengthened at the different levels; specific tasks were assigned and coordinated at each level; and demonstration sites were established for the communication approaches promoted in the workshops. Learning needs in the field of mass communication were also identified, and activities for training technical staff and for monitoring and evaluation were introduced in the national strategy 2018-2021 to guarantee the implementation and sustainability of communication activities. Notwithstanding, participating institutions will have to address the challenges characteristic of communication for arboviral disease prevention, which include the rotation of trained personnel, the mobilization of resources for communication, and the identification of appropriate evaluation indicators.

RESUMO O presente trabalho tem o objetivo de descrever as oportunidades e os desafios da capacitação em articulação de iniciativas regionais e nacionais de comunicação para prevenção de arboviroses, a partir dos ensinamentos tirados com a experiência cubana. Em fevereiro de 2016 e em dezembro de 2017, profissionais do Instituto de Medicina Tropical Pedro Kourí, em Cuba, participaram de um seminário sobre o fortalecimento da capacidade de comunicação para prevenção de arboviroses na atenção primária à saúde com a incorporação gradual dos enfoques mais recentes promovidos pela Organização Pan-Americana da Saúde (OPAS). Especialistas com diferentes perfis profissionais foram capacitados ao nível nacional, de província, do município e de áreas de saúde. Equipes multidisciplinares foram formadas e consolidadas nos diferentes níveis e elas receberam a incumbência de tarefas concretas coordenadas em cada nível e participaram da demonstração dos enfoques de comunicação apresentados nos seminários. Além disso, as necessidades de aprendizagem em comunicação social foram identificadas e atividades de capacitação do pessoal técnico e atividades de monitoramento e avaliação foram incorporadas à estratégia nacional 2018-2021 com o objetivo de garantir a implementação e a sustentabilidade das ações de comunicação. Porém, as instituições participantes terão de enfrentar os próprios desafios de comunicação na prevenção das arboviroses, por exemplo, rotatividade do pessoal capacitado, captação de recursos para comunicação e identificação dos indicadores adequados de avaliação.

Palavras-chave Comunicação social; Aedes aegypti; infecções por arbovirus; controle de vetores; Cuba. 